\title{
Effects of sevoflurane on leucine-rich repeat kinase 2-associated Drosophila model of Parkinson's disease
}

\author{
ZHIMING SHAN $^{1 *}$, SONG CAI $^{2 *}$, TAO ZHANG $^{1}$, LITING KUANG $^{1}$, QI WANG ${ }^{2}$, \\ HUANHUAN XIU $^{1}$, JING WEN ${ }^{1}$, HUAIYU GU $^{2}$ and KANGQING XU ${ }^{1}$ \\ ${ }^{1}$ Department of Anesthesiology, The First Affiliated Hospital of Sun Yat-sen University; \\ ${ }^{2}$ Department of Anatomy and Neurobiology, Zhongshan School of Medicine, \\ Sun Yat-sen University, Guangzhou, Guangdong 510080, P.R. China
}

Received June 24, 2014; Accepted September 24, 2014

DOI: $10.3892 / \mathrm{mmr} .2014 .2966$

\begin{abstract}
Patients with Parkinson's disease (PD) often require surgery, and therefore may receive inhalation anesthesia. However, it is currently unknown whether inhalation anesthetics affect the prognosis of the disease. Leucine-rich repeat kinase 2 (LRRK2) genetic mutations are the most common cause of familial PD, contributing to $39 \%$ of all cases in certain populations. The aim of the present study was to determine the effects of inhaled anesthetics on PD, by observing the influence of sevoflurane on a LRRK2-associated Drosophila model of PD. PD transgenic Drosophila overexpressing LRRK2 were generated by crossing flies expressing an LRRK2 upstream activation sequence, with tyrosine hydroxylase (TH)-Gal4 flies. Western blot analysis successfully verified that the transgenic Drosophila overexpressed LRRK2. Three days prior to eclosion, three genotypes of Drosophila were divided into four groups, and were exposed to air, 1, 2, or 3\% sevoflurane, for 5 hours. Twenty-four hours after the exposure, the electrophysiological activities of the projection neurons (PN) in the brains of the Drosophila were recorded using a patch clamp. The locomotor activities were tested on days 5, 10, 15, 20, 25, 30,35 and 40 following eclosion. The frequency of miniature excitatory synaptic currents (mEPSCs) obtained from the PNs of the TH-wild type LRRK2 (TH-WT) Drosophila
\end{abstract}

Correspondence to: Professor Kangqing $\mathrm{Xu}$, Department of Anesthesiology, The First Affiliated Hospital of Sun Yat-sen University, 58 Zhongshan Road, Guangzhou, Guangdong 510080, P.R. China

E-mail: szmraymond@163.com

Professor Huaiyu Gu, Department of Anatomy and Neurobiology, Zhongshan School of Medicine, Sun Yat-sen University, 74 Zhongshan Road, Guangzhou, Guangdong 510080, P.R. China E-mail: profgu@163.com

${ }^{*}$ Contributed equally

Key words: sevoflurane, Drosophila, LRRK2, Parkinson's disease, patch clamp brain, following exposure to air $(1.60 \pm 0.05 \mathrm{~Hz})$, was lower as compared with the wild type LRRK2 (WT) $(2.51 \pm 0.07 \mathrm{~Hz})$ and W1118 $(2.41 \pm 0.10 \mathrm{~Hz})$ Drosophila. After exposure to 1,2 and $3 \%$ sevoflurane, the frequency of mEPSCs in the brains of the TH-WT group decreased to $0.82 \pm 0.04 \mathrm{~Hz}, 0.63 \pm 0.16 \mathrm{~Hz}$ and $0.55 \pm 0.04 \mathrm{~Hz}$, respectively. The percentage decrease of the frequency of mEPSCs, from exposure to air to $1 \%$ sevoflurane, of the TH-WT group $(48.32 \% \pm 3.08 \%)$ was significantly higher, as compared with the WT $(39.17 \% \pm 1.42 \%)$ and W1118 $(35.10 \% \pm 2.66 \%)$ groups, and there was no statistical difference between the WT and W1118 groups. The transgenic TH-WT Drosophila presented an early decrease in locomotor ability, as compared with the WT and W1118 groups. Following a 5 hour exposure to sevoflurane, the percentage decrease of the climbing abilities of the TH-WT group, from exposure to air to $1 \%$ sevoflurane, were significantly lower, as compared with the WT and W1118 groups. In conclusion, sevoflurane had negative effects on the control W1118 flies, and also severely aggravated the prognosis of PD in the LRRK2-associated Drosophila model, through synaptic cholinergic deficits and impairment on locomotor abilities.

\section{Introduction}

Parkinson's disease (PD) is one of the most common neurodegenerative diseases that affects $>1 \%$ of the $>60$-year-old population, worldwide (1). An important pathophysiological mechanism of PD is associated with the progressive loss of nigrostriatal dopaminergic neurons (2). With an increasing number of elderly patients requiring surgical procedures, more patients with PD will receive general anesthesia (3).

Sevoflurane is an inhalational general anesthetic, with numerous beneficial properties, including low pungency, low blood/gas partition coefficient, rapid inhalation induction and recovery; sevoflurane, has therefore been widely used in clinical anesthesia (4). As the use of sevoflurane in general anesthesia has increased, there have been increasing concerns regarding the safety of inhalational anesthetics. Numerous studies have shown that persistent learning deficits and social behavior dysfunction of animals may occur, following exposure to general anesthesia $(5,6)$. The clinical concentration of sevoflurane has previously been shown to inhibit neurotransmission 
in a dose-dependent manner, as determined by a hippocampal slice study $(7,8)$. Mason et al (9) demonstrated that inhalational anesthetics may change the concentration of dopamine in the brain by impairing the synaptosomes, which mediate dopaminergic transmission. Such findings indicate that exposure to sevoflurane may affect dopaminergic neuronal function, and influence the disease. However, the effects of sevoflurane on patients with PD remains unknown.

The Drosophila melanogaster is an ideal model organism that is often used in the study of neuroscience complex biological function and disease research. Drosophila have an intact neural circuit and a specific anatomical structure that is beneficial for investigations into the mechanisms of neuropharmacology, neuropathology and biochemistry (10).

The leucine-rich repeat kinase 2 (LRRK2) gene, which is expressed in all examined tissues, spans a $144 \mathrm{~kb}$ genomic region, with 51 exons encoding 2,527 amino acids. A previous study treated mutations in the LRRK2 gene as a mature model of PD. LRRK2 mutations cause late-onset autosomal dominant PD with diverse pathologies, including the formation of Lewy bodies, nigral degeneration, and neurofibrillary tau-positive tangles (11). Liu et al (12) successfully generated a LRRK2-associated Drosophila model of PD, in order to verify that overexpression of LRRK2, which is one type of PD-associated LRRK2 genetic mutation, led to retinal degeneration, selective loss of dopamine (DA) neurons, and decreased climbing activity. DA is the only catecholaminergic neurotransmitter present in the central nervous system of Drosophila melanogaster, and it has an important role in the progression of PD (12).

Numerous studies have demonstrated that the loss of DA is a hallmark of PD pathology (14-16). In Drosophila, as well as vertebrates, tyrosine hydroxylase $(\mathrm{TH})$ catalyzes the rate-limiting step in DA biosynthesis, and the Drosophila TH gene has been shown to be specifically expressed in all dopaminergic cells (17). The present study crossed TH-Gal4 Drosophila with upstream activation sequence-wild type LRRK2 (UAS-WT) Drosophila, in order to generate transgenic Drosophila overexpressing LRRK2 specifically in dopaminergic cells.

In Drosophila, mushroom bodies are critical for associative learning and memory. Olfactory sensory neurons receive stimulation, and emit signals to the projection neurons (PNs), which are located in the antennal lobe. Then, PNs convey the olfactory signals to Kenyon cells, the principal cells of the mushroom body (18). As a paired neuropil structure in the central brain, the mushroom bodies are critical for associative learning and memory of Drosophila.

The PNs are cholinergic, and through the olfactory learning and memory circuit, nicotinic acetylcholine receptors are crucial factors for driving the majority of spontaneous excitation. Thus, patch clamp recordings of the miniature excitatory post synaptic currents (mEPSCs) of PNs can be used to evaluate cholinergic transmission in the Drosophila antennal lobe (D). Impairment of synaptic plasticity has previously been implicated in PD; therefore, the anomalous electrophysiological changes of PNs are associated with the synaptic transmission deficits that characterize neurodegenerative disease (20). The present study evaluated the synaptic functions of the brain using a patch clamp, that recorded electrophysiological signals of the PN from the antennal lobe.

\section{Materials and methods}

\section{Drosophila strains}

W1118 controls. W1118 Drosophila were used as controls for comparisons with the experimental mutants used in the present study. Both W1118 and experimental Drosophila were reared on standard cornmeal agar medium, supplemented with dry yeast, and maintained at $24^{\circ} \mathrm{C}$ in an atmosphere containing $60 \%$ relative humidity.

Mutants. The cDNA encoding wild-type LRRK2 was obtained from pcDNA3.1 (+) with BamHI/XhoI double digests, and cloned into the pUAST vector at the $B g l \mathrm{II} / \mathrm{XhoI}$ site (provided by Dr. Xicui Sun, Laboratory of Neurology, The First Affiliated Hospital of Sun Yat-sen University, Guangzhou, China). The plasmids were microinjected into W1118 Drosophila embryos (Genetic Services, Inc., Cambridge, MA, USA), in order to obtain UAS-WT LRRK2 Drosophila.

Cross. TH-Wild Type LRRK2 (TH-WT) transgenic Drosophila were generated using the GAL4/UAS system, as previously described (21), to overexpress LRRK2 protein, specifically in the dopaminergic cells of the brain.

Gender. In order to reduce the sampling bias, all Drosophila used in the present study were male.

Statement of Animal Care and Use Committee Approval. Animal handling and all experimental procedures used in the present study were approved by the Animal Care and Use Committee of Sun Yat-sen University (Guangzhou, China), and were conducted in accordance with the National Institutes of Health (NIH) Guide for the Care and Use of Laboratory Animals (NIH, Bethesda, MA, USA).

Western blot analysis. The heads of the adult Drosophila were collected and homogenized in lysis buffer $(50 \mathrm{mM}$ Tris-HCl, pH 7.5/1 mM EDTA/0.5 M NaCl/1\% Triton $\mathrm{X}-100 / 1 \mathrm{mM}$ DTT with protease inhibitors) at $4^{\circ} \mathrm{C}$ for $15 \mathrm{~min}$. After centrifuging at $12,000 \mathrm{x} \mathrm{g}$ for $10 \mathrm{~min}$ at $4^{\circ} \mathrm{C}$, the supernatants were loaded onto SDS-PAGE gels, separated by electophoresis and then transferred to polyvinylidene fluoride membranes $(0.45 \mathrm{~mm}$, EMD Millipore, Billerica, MA, USA). The membranes were blocked with TBST ( $\mathrm{pH} 7.4,10$ $\mathrm{mM}$ TrisHCl/150 mM NaCl/0.1\% Tween 20) containing 5\% fat-free milk for $2 \mathrm{~h}$, then probed by incubation with a monoclonal mouse ANTI-FLAG ${ }^{\circledR}$ M2 antibody at a 1:1,000 dilution (F3165; Sigma-Aldrich, St Louis, MO, USA), overnight at $4^{\circ} \mathrm{C}$. The membranes were then incubated with a polyclonal horseradish peroxidase (HRP)-conjugated goat-anti-mouse immunoglobulin G secondary antibody (A3682; SigmaAldrich), at a 1:10,000 dilution. Chemiluminescent HRP substrate (EMD Millipore) was used to detect the HRP, and the blots were visualized by exposure to Kodak MR film (Kodak, Rochester, NY, USA). The membranes were then stripped and reprobed with mouse anti- $\beta$-actin antibody (sc-1616-R Santa Cruz) 1:4,000 dilution. $\beta$-actin was used as a loading control. All western blot tests were performed three times. All results have been quantified and analyzed with Image $\mathrm{J}$ software version 1.46r (National Institutes of Health Bethesda, MA, USA). 
Sevoflurane exposure. All of the experimental groups were exposed to sevoflurane three days prior to eclosion. The sevoflurane groups $(1,2$, or $3 \%$ sevoflurane exposure for $5 \mathrm{~h})$ were placed in a special anesthesia glass box, produced by the laboratory of Anatomy and Neurology, (Zhongshan School of Medicine, Sun Yat-sen University). The box measured $200 \mathrm{~mm} \times 200 \mathrm{~mm} \times 100 \mathrm{~mm}$ with a hole set in the middle of the upper face for the delivery of gaseous anaesthetics via an anesthesia machine. Air was used as a carrier and the airflow was controlled at $2 \mathrm{l} / \mathrm{min}$. The levels of sevoflurane, $\mathrm{O}_{2}$ and $\mathrm{CO}_{2}$ in the chamber were monitored using a gas monitor (GE Healthcare, Chalfont, UK), which displayed the instantaneous gas concentration on an LCD monitor. The temperature in the experimental room was controlled at $24^{\circ} \mathrm{C}$ with $60 \%$ humidity. The control groups received air without sevoflurane. Twenty-four hours after $5 \mathrm{~h}$ anesthetic exposure, the anesthesia groups were subjected to subsequent experimental procedures.

Electrophysiology. The brains were obtained from the Drosophila two days prior to eclosion, 24 hours after sevoflurane or air exposure. The entire brain, including the optic lobes, was removed from the head and prepared for recordings in a standard external solution containing $20 \mathrm{units} / \mathrm{ml}$ papain, with $1 \mathrm{mM}$ L-cysteine, as previously described $(22,23)$. The standard external solution contained (in $\mathrm{mM}$ ): $101 \mathrm{NaCl}, 1 \mathrm{CaCl}_{2}$, $4 \mathrm{MgCl}_{2}, 3 \mathrm{KCl}, 5$ glucose, $1.25 \mathrm{NaH}_{2} \mathrm{PO}_{4}$, and $20.7 \mathrm{NaHCO}_{3}$, $\mathrm{pH} 7.2$, Osm 250. The dissected brains were then mounted in an RC-26 perfusion chamber (Warner Instruments, Hamden, CT, USA), and the recording solution was bubbled through with $95 \% \mathrm{O}_{2}$ and $5 \% \mathrm{CO}_{2}(2 \mathrm{ml} / \mathrm{min})$ during the experiment, with the anterior brain facing up. Pipettes were targeted to PNs in the dorsal neuron cluster of the antennal lobe.

Whole-cell recordings were performed with pipettes (10-15 M 2 ) filled with an internal solution containing the following (in $\mathrm{mM}$ ): $102 \mathrm{~K}$-gluconate, $0.085 \mathrm{CaCl}_{2}, 1.7 \mathrm{MgCl}_{2}$, $17 \mathrm{NaCl}, 0.94$ EGTA, and 8.5 HEPES with $\mathrm{pH} 7.2$ and 235 mOsm. TTX was used to block sodium channels and PTX was used to block GABA receptors as previously described (24). The pipette solution for calcium and sodium currents (in $\mathrm{mM}$ ) consisted of: $102 \mathrm{D}$-gluconic acid, $102 \mathrm{CsOH}, 0.085 \mathrm{CaCl}_{2}$, $1.7 \mathrm{MgCl}_{2}, 17 \mathrm{NaCl}, 0.94$ EGTA and 8.5 HEPES, and $\mathrm{pH} 7.2$, $235 \mathrm{Osm}$.

Voltage-clamp recordings were performed using borosilicate glass electrodes (B150-86-10; Sutter Instrument Co., Novato, CA, USA) as previously described (24). Gigaohm seals were achieved prior to recording the on-cell configuration, followed by the whole-cell configuration, whilst in the voltage-clamp mode. The recordings were made at room temperature, and only a single PN was examined in each brain.

All electrophysiological recordings were carried out using a BX51WI upright microscope (Olympus, Center Valley, PA, USA). The signals were acquired using an EPC10 amplifier (HEKA Elektronik, Lambrecht/Pfalz, Germany) and were filtered at $5 \mathrm{kHz}$, using a built-in filter digitized at $5 \mathrm{kHz}$. Data analysis was performed using the MiniAnalysis 6.0 program (Synaptosoft, Inc., Fort Lee, NJ, USA).

Climbing ability. All pupae were incubated in test tubes. After being anesthetized, a number of the pupae were selected for electrophysiological experiments, and the remainder were incubated in the tube until eclosion. Following eclosion, the flies were exposed to sevoflurane or air, and collected in order to perform climbing assays, on days $5,10,15,20,25,30,35$ and 40 . A total of 10 flies were placed in empty glass vials $(10.5 \mathrm{~cm} \times 2.5 \mathrm{~cm})$. A horizontal red line was drawn $8 \mathrm{~cm}$ above the bottom of the vial. The Drosophila were allowed to accommodate to the vials for $10 \mathrm{~min}$ at room temperature, after which both the control and experimental groups were assayed randomly, in a series of 10 repetitive trials for each. Before each trial, the vials were gently tapped, in order for the Drosophila to remain at the bottom of the vial. The number of Drosophila above the red line of the vial was counted after $10 \mathrm{sec}$ of climbing, and the same batch of Drosophila was used to repeat the trials 10 times. The values obtained were then averaged, and a group mean and standard error were calculated. The mean values for the various groups were statistically compared, using an unpaired student's t-test. All behavioral studies were performed at $24^{\circ} \mathrm{C}$, under standard lighting conditions.

Statistical analyses. The data are presented as the means \pm standard deviation. Comparisons between the groups were performed using a one way analysis of variance, followed by a Bonferroni-Dunn post hoc test or independent sample tests. All of the electrophysiological data were analyzed using the MiniAnalysis 6.0 program (Synaptosoft Inc.). A P $<0.05$ was considered to indicate a statistically significant difference.

\section{Results}

Generation of LRRK2 transgenic Drosophila. Overexpression LRRK2 mutations are the most common cause of familial PD, contributing to $\sim 39 \%$ of all cases in certain populations (25). To create a model for the LRRK2-linked disease, transgenic Drosophila carrying a full-length WT LRRK2 gene were generated. The WT group was obtained by microinjecting a vector that contained UAS-LRRK2 into W1118 embryos. A Ga14/UAS bipartite system was used to ectopically express the transgenes. This system took advantage of the yeast GAL4 transcription factor, by binding specifically to the UAS. Therefore, UAS-linked transgenes were expressed in specific cells, under the control of a given promoter (promoter-GAL4). $\mathrm{TH}$ is an enzyme that catalyzes the rate-limiting step in DA biosynthesis, and is specific to dopaminergic cells (26). Therefore, TH-Gal4 was used as a promoter, in order to generate flies which specifically overexpressed the LRRK2 protein on dopaminergic cells (TH-WT). A western blot analysis was conducted to confirm the overexpression of LRRK2 in the TH-WT group. A strong band was observed at $>250 \mathrm{kDa}$ in the blots from the TH-WT group, that was not present in the control W1118 or WT groups (Fig. 1). These results indicate that only TH-WT Drosophila expressed the LRRK2 transgenes.

Confocal images. Confocal images of the Drosophila brain (Fig. 2A) showed the detailed morphology of the olfactory PN, as labeled with biocytin. In the olfactory learning and memory system of the Drosophila, the olfactory receptor neurons expressed the same odorant receptor that projected their axons to the same glomerulus in the antennal lobe. PNs 


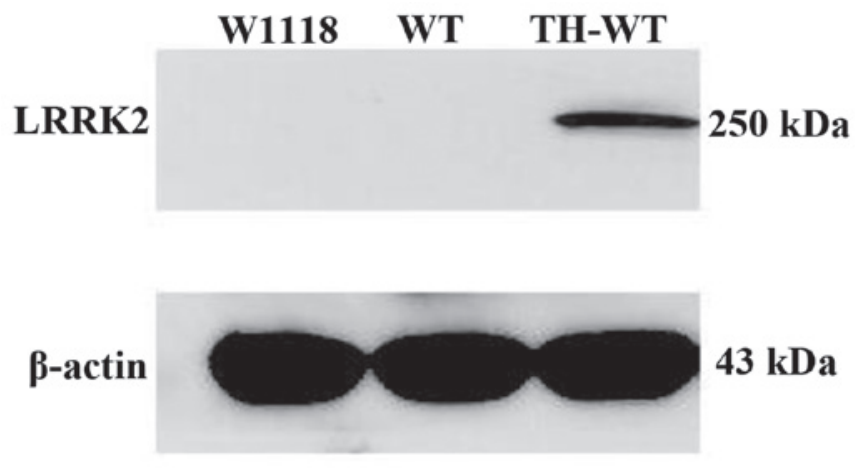

Figure 1. Protein expression levels of leucine-rich repeat kinase 2 (LRRK2) in flies containing the tyrosine hydroxlase (TH)-GAL4 promoter, in combination with the wild type transgene LRRK2 upstream activation sequence. Head extracts from the indicated Drosophila stocks were subjected to western blot analysis, using anti-FLAG ${ }^{\circledR}$ antibodies. TH-WT, tyrosine hydroxylase-wild type flies; WT, wild-type flies; W118, control flies; kDa, kilodaltons.

sent olfactory information through their axons, to the mushroom body and lateral horn. In the absence of stimuli, PNs continued to receive a continuous and spontaneous barrage of excitatory postsynaptic potentials (EPSPs), and spikes recorded in the PN somata were $<10 \mathrm{mV}$ (Fig. 2B). A nicotinic acetylcholine receptor antagonist blocked the spontaneous EPSPs in the PNs, and the spikes were blocked by TTX. The morphology and identity of a recorded neuron was confirmed by injecting with $0.4 \%$ biocytin in the recording pipette for at least $30 \mathrm{~min}$ in the whole cell configurations. After electrophysiological recording, the brain was fixed in phosphate buffered $4 \%$ formaldehyde at $4^{\circ} \mathrm{C}$ for $10 \mathrm{~h}$ and then washed in $1 \%$ PBS three times, blocked and incubated in blocking buffer (0.1 MPBS, $0.1 \%$ Triton X-100, $1 \%$ BSA) containing streptavidin-CY3 (Molecular Devices) for $3 \mathrm{~h}$ at room temperature, followed by three washes at $5 \mathrm{~min}$ intervals in PBS. A confocal microscope (LSM 710, Zeiss, Jena Germany) with a $\mathrm{x} 40$ objective was used to acquire photos of dendritic arborization of the visual projection neurons.

Electrophysiological recordings. A patch clamp was used to record the electrophysiological effects of sevoflurane on the frequency and amplitude of mEPSCs of PNs, in the isolated Drosophila pupa brain of the various genotypes, $24 \mathrm{~h}$ following exposure to sevoflurane or air. Cholinergic mEPSCs were obtained in the presence of $1 \mu \mathrm{mol} \mathrm{TTX}$ and $50 \mu \mathrm{mol}$ pertussis toxin. The standard characteristics of spontaneous postsynaptic currents and mEPSCs are compared in Fig. 3A.

The frequency of mEPSCs was compared between the three Drosophila genotypes, following exposure to air. The frequency of mEPSCs was markedly lower in the TH-WT group $(1.60 \pm 0.05 \mathrm{~Hz})$, as compared with the WT $(2.51 \pm 0.07 \mathrm{~Hz})$ and W1118 $(2.41 \pm 0.10 \mathrm{~Hz})$ groups. The frequency of mEPSCs in the WT group was not significantly different, as compared with the W1118 group (Fig. 3B). These results demonstrate that without the presence of the TH-Gal4 promoter the mutant UAS-LRRK2 gene had no effect on the frequency of mEPSCs in the PNs of the Drosophila. The significant decrease in the frequency of mEPSCs of PNs in the TH-WT group may be attributed to the successful overexpression of the LRRK2 protein in dopaminergic cells.
The effects of sevoflurane on normal and transgenic Drosophila is currently unknown. Therefore, in the present study W118, WT and TH-WT Drosophila were exposed to various concentrations of sevoflurane for $5 \mathrm{~h}$. In response to all three concentrations of sevoflurane, the frequency of mEPSCs of PNs in the normal W1118 Drosophila was significantly decreased $(1.54 \pm 0.05 \mathrm{~Hz}$ in $1 \%, 1.05 \pm 0.06 \mathrm{hz}$ in $2 \%$, and $0.97 \pm 0.05 \mathrm{~Hz}$ in $3 \%$ sevoflurane), as compared with the control W1118 group, which was exposed to air $(2.41 \pm 0.10 \mathrm{~Hz}$; Fig. 3B) These results indicate that sevoflurane may decrease the frequency of mEPSCs of PNs in the normal W1118 Drosophila.

The frequency of mEPSCs in the transgenic Drosophila were also significantly decreased, following exposure to sevoflurane. The frequency of mEPSCs in the WT group $(1.55 \pm 0.04 \mathrm{~Hz}$ in $1 \%, 1.11 \pm 0.04 \mathrm{~Hz}$ in $2 \%$, and $1.00 \pm 0.10 \mathrm{~Hz}$ in $3 \%$ sevoflurane) was much lower, as compared with the WT group that was exposed to air $(2.51 \pm 0.07 \mathrm{~Hz})$. In the TH-WT group, the decreased frequency of mEPSCs was more evident $(0.82 \pm 0.04 \mathrm{~Hz}$ in $1 \%, 0.63 \pm 0.16 \mathrm{~Hz}$ in $2 \%$, and $0.55 \pm 0.04 \mathrm{~Hz}$ in $3 \%$ sevoflurane), as compared with the group exposed to air $(1.60 \pm 0.05 \mathrm{~Hz}$; Fig. 3B). These results suggest that sevoflurane may decrease the frequency of mEPSCs, not only in normal W1118 Drosophila, but also in transgenic WT and TH-WT Drosophila.

In order to further investigate the various effects of sevoflurane on the three Drosophila groups, the percentage decrease of the frequency of mEPSCs in the flies, following exposure to sevoflurane, was determined. A concentration of $1 \%$ sevoflurane was considered to be effective, since all three groups presented significant decreases in the frequency of mEPSCs of PNs following exposure to $1 \%$ sevoflurane. In addition, there were no statistical differences between $1 \%$ sevoflurane and the other concentrations used. The percentage decrease was compared between the groups exposed to air and $1 \%$ sevoflurane. The decreased frequency of mESPCs in the TH-WT flies $(48.32 \% \pm 3.08 \%)$ was significantly higher, as compared with the WT $(39.17 \% \pm 1.42 \%)$ and W1118 groups $(35.10 \% \pm 2.66 \%)$. There was no statistical difference between the WT and W1118 groups (Fig. 3C).

Furthermore, there was no significant difference between the amplitude of mEPSCs among the different genotypes, and the groups treated with different concentrations of sevoflurane (Fig. 3D). These findings indicate that sevoflurane could affect normal W1118 flies by decreasing the frequency of mEPSCs of PNs; however, this effect was more severe in the TH-WT flies with PD.

Locomotor activity. PD is a movement disorder; therefore, the present study investigated how LRRK2 overexpression affected locomotor ability of the transgenic Drosophila, with or without anesthesia. A climbing assay, which has been previously used in transgenic Drosophila models of PD, was performed to assess locomotor activity. After a single exposure to sevoflurane or air, the Drosophila were collected in order to perform the reduplicative climbing assays, at various time points. The climbing ability of the W1118 and WT groups remained essentially unchanged up to 30 days, but significantly decreased following this time point (Fig. 4A). This finding is concordant with the results of a previous report, by Feany and 
A

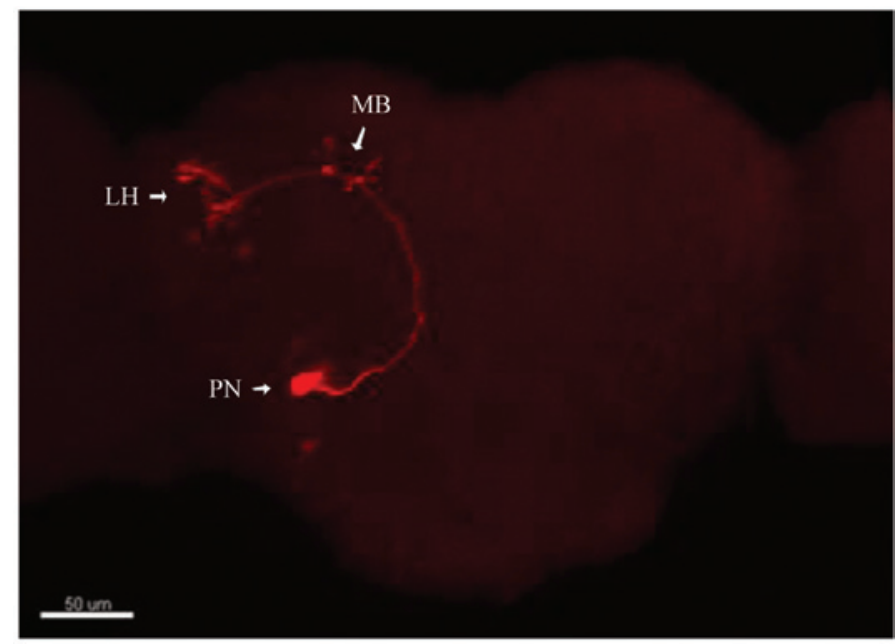

B

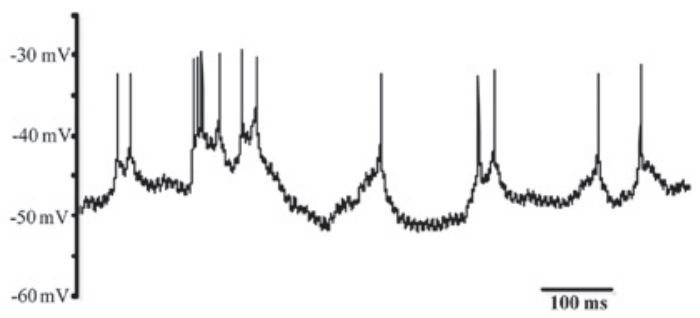

Figure 2. (A) Confocal image (magnification, $\mathrm{x} 40$ ) of the Drosophila brain showing the detailed morphology of the olfactory projection neuron (PN), labeled with biocytin (red). Typically, in the dorsal antennal lobe glomeruli where the dendritic aborization is located, the soma stalk of the PN sends out a large axon bundle which then develops into small collaterals, extending anteriorly and projecting to the mushroom body (MB) and lateral horn (LH). The scale bar represents $50 \mu \mathrm{m}$. (B) Spontaneous activity was recorded in the soma of the PN. The average recordings from the spikes in the PN somata were $<10 \mathrm{mV}$. Ms, milliseconds; $\mathrm{mV}$, millivolts.

A

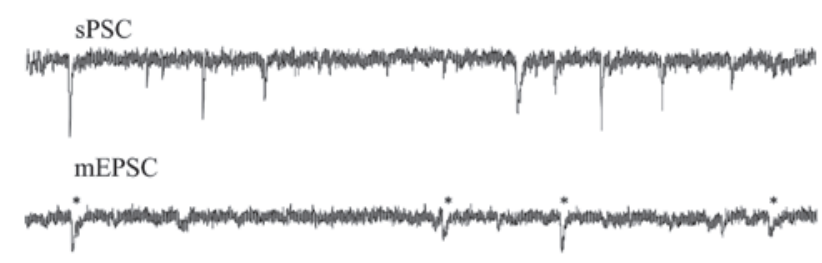

C

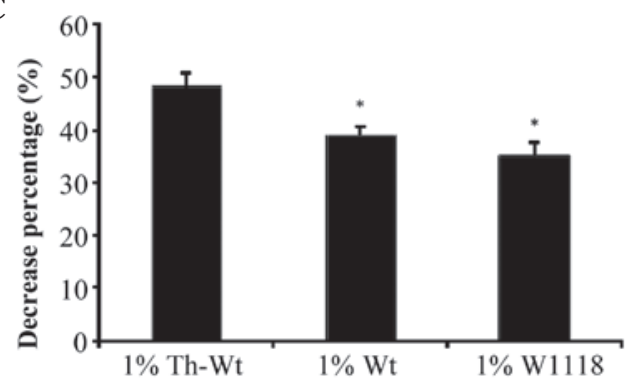

B

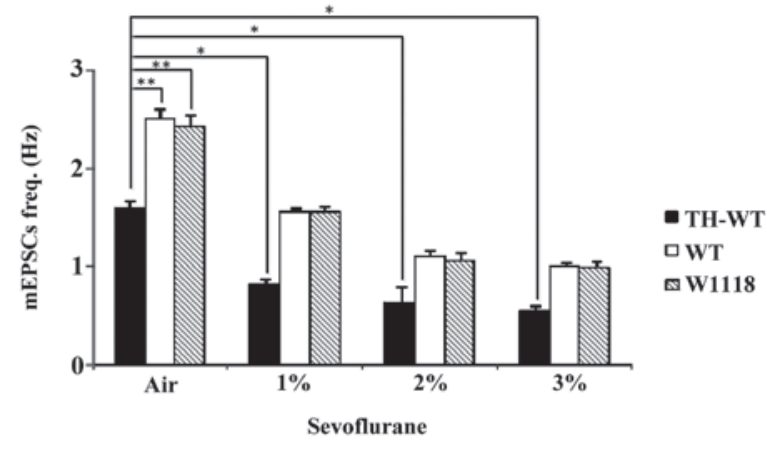

D

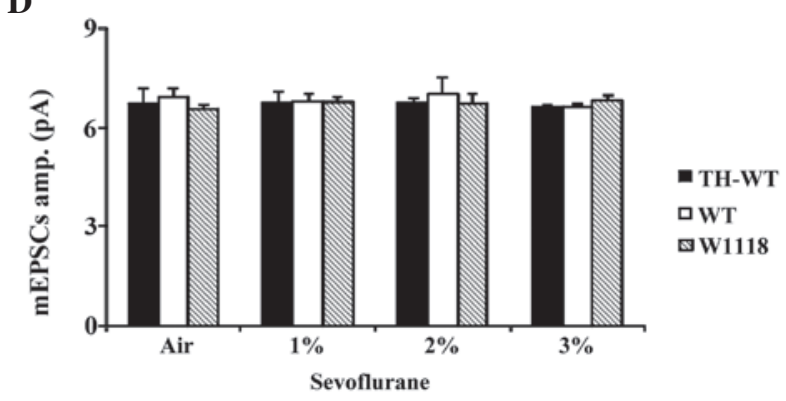

Figure 3. Miniature excitatory synaptic currents (mEPSCs) obtained from projection neurons (PNs) of the Drosophila brain of the three different genotypes, treated with various concentrations of sevoflurane. (A) Representative spontaneous postsynaptic currents (sPSCs) and mEPSCs were recorded from PNs of the wild type (WT) Drosophila brains. The mEPSCs wree obtained in the presence of tetrodotoxin and pertussis toxin, "standard mEPSC. (B) The mean frequency of mEPSCs in the tyrosine hydroxylase (TH)-WT, WT and W1118 Drosophila, under normal conditions and treated with sevoflurane at various concentrations. A Bonferroni post hoc test was used to determine significance. ${ }^{* *} \mathrm{P}<0.01 ;{ }^{*} \mathrm{p}<0.05$. (C) The percentage decrease of the frequency of mEPSCs, following treatment with $1 \%$ sevoflurane. $\mathrm{P}<0.05$. (D) The mean amplitude of mEPSCs in TH-WT, WT and W1118 Drosophila under normal conditions and following treatment with sevoflurane at various concentrations. TH-WT, tyrosine hydroxylase-wild type Drosophila; WT, wild-type Drosophila; W118, control Drosophila; Hz, hertz; freq, frequency; amp, amplification. 
Bender (27). The transgenic TH-WT group presented equivalent climbing ability to the W1118 and WT groups within 15 days. However, between days 20 and 40, the climbing abilities of the transgenic TH-WT group gradually decreased, as compared with the W1118 and WT groups ( $\mathrm{P}<0.05$, Fig. 4A). These results indicate that overexpression of LRRK2 in dopaminergic cells reduced the locomotor abilities of the TH-WT flies. This reduction was detected in the climbing assay 20 days following eclosion. However, there was no statistically significant difference in the climbing abilities of the WT, as compared with the W1118 Drosophila, from day 1 to 40 after eclosion. These data suggest there were no observable effects on locomotor ability during this time course, following LRRK2 cDNA microinjection into the W1118 Drosophila embryos, other than overexpression of LRRK2 protein.

An electrophysiological recording was conducted to compare the effects of the three different concentrations of sevoflurane. A concentration of $1 \%$ sevoflurane was considered to be effective. In the climbing assay experiment, $1 \%$ sevoflurane was regarded as the effective concentration, for comparing the results with the group exposed to air. In the normal W1118 Drosophila there were no differences in the locomotor abilities, between the experimental and control groups, until 25 days after eclosion (Fig. 4B). However, following the exposure to $1 \%$ sevoflurane, the WT flies had significantly decreased locomotor ability, from day 25-35, as compared with the control group (Fig. 4C). This decrease was even more evident in the transgenic TH-WT group, in which a significant difference was observed between days 10 and 40 (Fig. 4D). In conclusion, sevoflurane not only decreased the locomotor abilities of the normal W1118 and WT Drosophila, but also deteriorated the climbing capacities of the transgenic TH-WT Drosophila.

In order to determine the difference in the effects of sevoflurane on the W1118 and transgenic Drosophila, the percentage decrease of the climbing abilities was compared between the W1118, WT and TH-WT groups, with or without exposure to $1 \%$ sevoflurane. Notably, from day $10-40$, the percentage decrease of the climbing abilities of the TH-WT group was significantly lower, as compared with the WT and W1118 groups. The percentage decrease of the WT Drosophila remained similar to that of the W1118, except at day 35. These comparisons indicate that sevoflurane led to a more severe deterioration of locomotor ability in the TH-WT, as compared with the WT and W1118 flies (Fig. 4E).

\section{Discussion}

Genetic mutations of LRRK2 are considered to be the most common known genetic cause of familial PD, with a similar clinical progression and neurochemical genotype to typical late-onset disease. In the present study, in order to acquire a Drosophila model with typical PD characteristics, TH-Gal4 was used as a promoter to specifically induce LRRK2 overexpression in DA neurons. Previous studies have successfully established a Drosophila model of PD using the Gal4/UAS system, to generate transgenic Drosophila overexpressing wild-type LRRK2 (12). Based on the various types of Gal4 promoter, researchers may specifically overexpress LRRK2 in any cell type, depending on their requirements. DA neurons have important roles in the pathogenesis of $\mathrm{PD}$, and the over- expression of LRRK2 in DA neurons can lead to severe DA lesions and apoptosis (13). A previous study demonstrated that overexpression of LRRK2 in all neurons, under the control of the pan neuronal promoter elav-GAL4, lead to a less severe genotype in flies, as compared with those specifically overexpressing LRRK2 in DA neurons. However, the protein expression levels were higher in the head homogenates of the elav-GAL4 LRRK2 Drosophila. LRRK2 triggers the loss of anti-TH immunostaining; however, there is no significant loss in anti-elav or anti-5-HT immunostaining (28-30). These findings may explain why the protein expression levels of LRRK2 in DA neurons are reduced by elav-GAL4, indicating that LRRK2-induced toxicity is preferentially localized to DA neurons in the brain, which is concordant with human PD. TH catalyzes the rate-limiting step in DA biosynthesis, therefore the Drosophila TH gene is specifically expressed in all dopaminergic cells (17).

As previously reported, mEPSCs of PNs in the Drosophila central nervous system are associated with synaptic stability and plasticity (31). Therefore, mEPSCs may have a critical role in the functional and structural aspects of the synapses of PNs (32). Talantova et al (33) previously demonstrated that a decreased frequency of mEPSCs may cause early synaptic injury, due to concurrent extrasynaptic N-methyl-D-aspartare receptor-mediated nitric oxide production, tau phosphorylation, and caspase- 3 activation. In the present study, the frequency of mEPSCs in the PNs of transgenic TH-WT Drosophila brains was significantly decreased, following exposure to air, as compared with the W1118 and WT groups. These results suggest that the synaptic transmission of Drosophila with PD was lower, as compared with the normal Drosophila. The frequency of mEPSCs of PNs in the transgenic TH-WT Drosophila may be declined due to disorder of the dopamine-cholinergic system.

In addition, mEPSCs are thought to be involved in synaptic plasticity in the Drosophila central nervous system (32-34). mEPSCs are evoked by single vesicle release, which is triggered by release of presynaptic calcium ions (35). Alterations to the frequency and amplitude of mEPSCs are ascribed to presynaptic and postsynaptic action (36). The amplitude of mEPSCs reflects the response of the postsynaptic receptor to a single vesicle, while the frequency is partially due to changes to the presynaptic calcium channel (35). In the present study, following exposure to various concentrations of sevoflurane, the frequency of mEPSCs of PNs in the normal W1118 flies was significantly decreased. These results indicate that sevoflurane may affect normal W1118 Drosophila by reducing the presynaptic calcium channels of PNs. Conversely, the amplitude of mEPSCs remained the same. Therefore, the effects of sevoflurane on postsynaptic action are not as evident as they are on presynaptic calcium ions. Furthermore, the WT and TH-WT groups exhibited a similar decrease in the frequency of mEPSCs of PNs, as compared with the W1118 group. However, the differences of the negative effects between the three concentrations of sevoflurane on the frequency of mEPSCs were not as significant as initially predicted. Therefore, $1 \%$ sevoflurane was considered to be an effective concentration and was used for all further experiments.

Percentage decreases were calculated by comparing the frequency of mEPSCs in the Drosophila exposed to $1 \%$ sevo- 
A

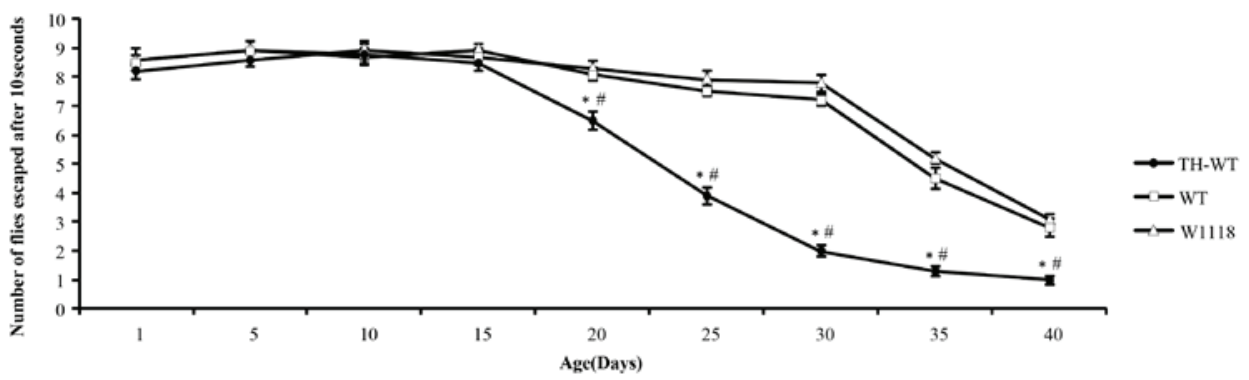

B

W1118

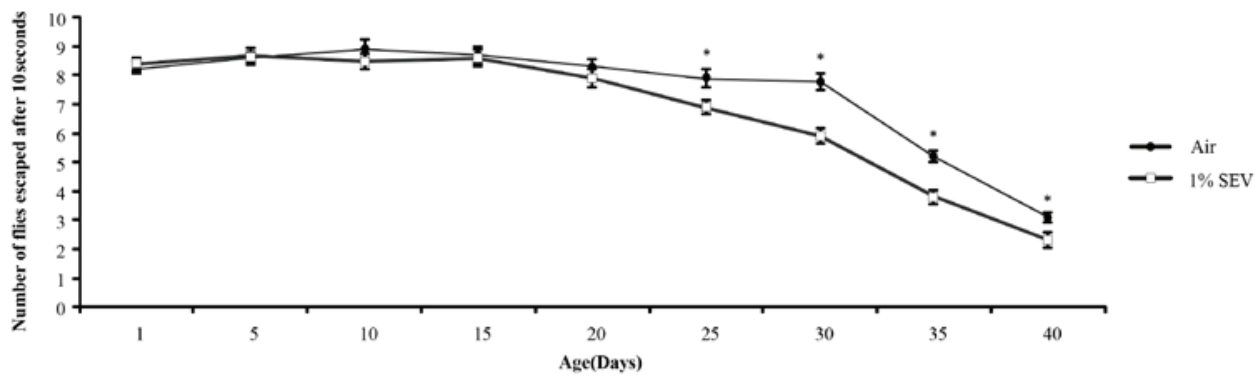

C

WT

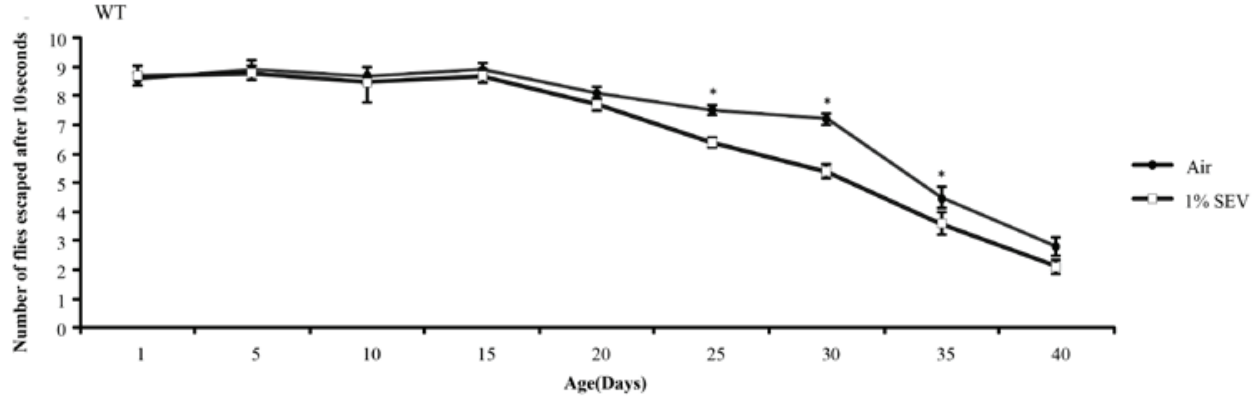

D

TH-WT

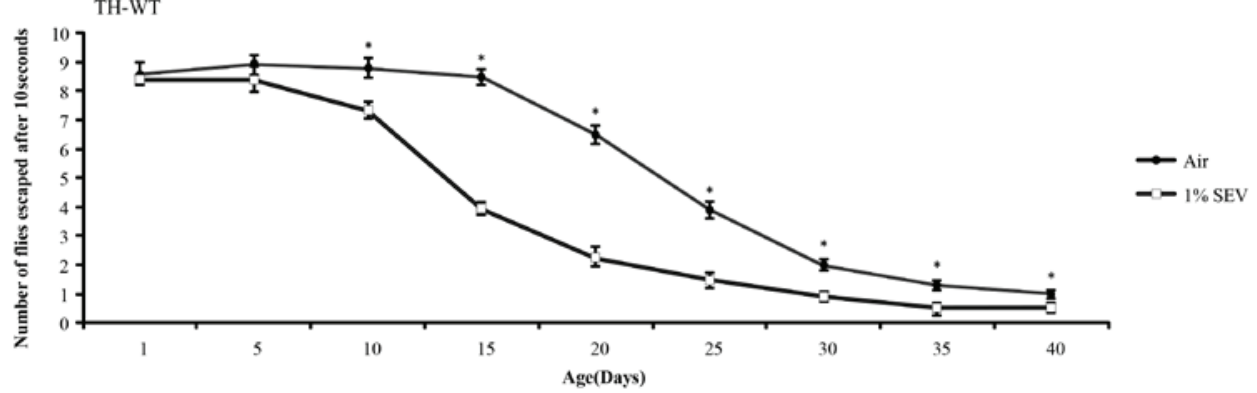

$\mathbf{E}$

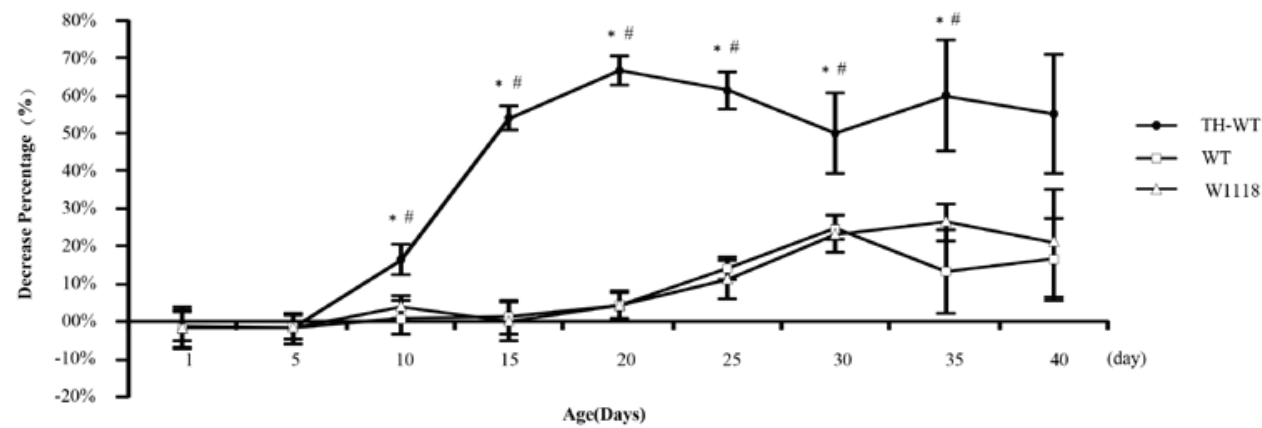

Figure 4. Sevoflurane exposure to the three genotypes of Drosophila, resulted in various alterations to locomotor activity. Each data point represents a group of 10 Drosophila, which underwent 10 replicated assays, to calculate the means and error bars. (A) Climbing ability of the transgenic and control Drosophila, from day 1-40, without sevoflurane exposure. " $\mathrm{P}<0.05$, tyrosine hydroxylase-wild type (TH-WT) vs WT Drosophila; ${ }^{\sharp} \mathrm{P}<0.05$, TH-WT vs W1118 Drosophila. (B) Climbing ability of the W1118 Drosophila following exposure to air and $1 \%$ sevoflurane; ${ }^{*} \mathrm{P}<0.05$. (C) Climbing ability of the WT Drosophila following exposure to air and $1 \%$ sevoflurane; " $\mathrm{P}<0.05$. (D) Climbing ability of the TH-WT Drosophila following exposure to air and $1 \%$ sevoflurane; $\mathrm{P}<0.05$. (E) Percentage decrease of the three types of Drosophila, following exposure to $1 \%$ sevoflurane. ${ }^{*} \mathrm{P}<0.05$, TH-WT vs WT Drosophila; ${ }^{~} \mathrm{P}<0.05$, TH-WT vs W1118 Drosophila. All of the data were analyzed by a one way analysis of variance, followed by a Bonferoni's post hoc test. TH-WT, tyrosine hydroxylase-wild type Drosophila; WT, wild-type Drosophila; W118, control Drosophila. 
flurane, with the Drosophila exposed to air. The percentage decrease of TH-WT group $(48.32 \% \pm 3.08 \%)$ was significantly higher, as compared with the WT $(39.17 \% \pm 1.42 \%)$ and W1118 groups $(35.10 \% \pm 2.66 \%)$, and there was no statistical difference between the WT and W1118 groups. These results indicate that sevoflurane may cause more severe effects on Drosophila with PD. The possible mechanism of anesthetic-induced PD related mEPSC impairment may be due to the potential effectss of the anesthetics, on synaptic morphology and function. It may be hypothesized that sevoflurane may regulate synaptic developmental processes and modulate aberrant synaptic formation or ectopic neuron distribution, leading to impairment of synaptic plasticity and maturation, which have already been damaged by the progression of PD (37). In the present study, sevoflurane markedly reduced the frequency of mEPSCs of Drosophila with PD, and this may be due to the basic impairment of synaptic plasticity implicated in PD.

Movement disorder is a common symptom of patients with PD (38). As compared with the immediate effects observed on mEPSCs, the effects of LRRK2 overexpression on locomotor behavior are chronic, which become more significant as the Drosophila age (39). Furthermore, the life span of the W1118 Drosophila is $\sim 45.5$ days following eclosion (40); however, it is even longer in the transgenic LRRK2 Drosophila (25). As a result, the locomotor abilities of the Drosophila were assessed between days 1 and 40 following eclosion. The climbing abilities of the W1118 and WT groups remained unchanged before 30 days, but gradually declined thereafter. This finding indicates that, without being activated by TH-Gal4, the locomotor activities of both the WT and W1118 Drosophila were identical. A vector containing UAS-WT LRRK2 with a TH-Gal4 promoter resulted in the successful generation of TH-WT Drosophila with an overexpression of the LRRK2 protein. During the climbing assay, the climbing abilities of the TH-WT group were significantly decreased from day 20-40 following eclosion, indicating that the overexpression of LRRK2 led to locomotor impairment in the Drosophila after day 20. Previous studies have demonstrated that such impairment is associated with the loss of DA neurons in the brain $(41,42)$. Therefore, it may be speculated that locomotor impairment of the Drosophila overexpressing LRRK2, specifically in the DA neurons, may result from the loss of dopaminergic transmission.

In the present study, the climbing abilities of the W1118 Drosophila following exposure to $1 \%$ sevoflurane were significantly decreased from day 25-35, suggesting that sevoflurane could deteriorate the locomotor ability of the W1118 Drosophila; however, such deterioration could only be distinguished 25 days following eclosion. After exposure to sevoflurane during pupa, the synaptosomes in the brains of the Drosophila may deteriorate, resulting in deficits to locomotor ability. Deterioration also occurred in the transgenic WT group, that was quite similar to that of the W1118 group.

As Mason et al (9) previously suggested, inhalational anesthetics may change the concentration of DA in the brain by impairing the synaptosomes that mediate dopaminergic transmission; therefore, it may be deduced that the negative effects of sevoflurane could be found in patients with PD. In the present study, sevoflurane appeared to cause markedly severe damage to the locomotor ability of TH-WT
Drosophila, whose climbing ability significantly decreased from day 10. In order to verify the differences in the effects of sevoflurane between the W1118 and transgenic groups, the percentage decreases were compared between the W1118, WT and TH-WT, which were calculated by comparing the frequency of mEPSCs of the Drosophila exposed to $1 \%$ sevoflurane, with the Drosophila exposed to air. Notably, between days 10 and 40, the percentage decrease of the climbing abilities of the TH-WT group was significantly lower, as compared with the WT and W1118 groups. These comparisons indicate that sevoflurane led to a more severe deterioration in the locomotor ability of the TH-WT, as compared with the WT and W1118 groups. Overexpression of LRRK2 in dopaminergic cells leads to neurofibrillary tau-positive tangles, nigral degeneration, Lewy bodies and specific loss of dopaminergic neurons in the aging brain; which may be the cause of the deteriorating locomotor abilities in the Drosophila overexpressing LRRK2, 15 days after eclosion $(43,44)$. Sevoflurane increases brain extracellular concentrations of DA during general anesthesia by impairing the transport synaptosomes of DA. Such mechanisms may lead to further damage of the dopaminergic system and locomotor dysfunction (9).

In conclusion, sevoflurane not only had negative effects on normal W1118 Drosophila, but also severely aggravated the prognosis of PD in a LRRK2-associated Drosophila model, by means of synaptic cholinergic deficits and impairment of locomotor abilities. The possibility of other impairments in the prognosis of PD by sevoflurane, however, remain uncertain. There are also doubts as to whether sevoflurane will have the same effects on humans with PD. Therefore, further investigations are required in order to fully understand the effects of sevoflurane on patients with PD.

\section{Acknowledgements}

The authors of the present study would like to thank Xicui Sun, Peisen Huang and Runcong Nie for instructions regarding the successful creation of a cross-transgenic model. They would also like to acknowledge the help of Youlan Li in proofreading the manuscript and data analysis. This study was supported by a grant from the Guangdong Science Foundations (grant no. S2011010003739).

\section{References}

1. Nicholson G, Pereira AC and Hall GM: Parkinson's disease and anaesthesia. Br J Anaesth 89: 904-916, 2002.

2. Belin AC and Westerlund M: Parkinson's disease: a genetic perspective. FEBS J 275: 1377-1383, 2008.

3. García-Pérez L, Linertová R, Lorenzo-Riera A Vázquez-Díaz JR, Duque-González B, Sarría-Santamera A: Risk factors for hospital readmissions in elderly patients: a systematic review. QJM 104: 639-651, 2011

4. Zhang X, Xue Z and Sun A: Subclinical concentration of sevoflurane potentiates neuronal apoptosis in the developing C57BL/6 mouse brain. Neurosci Lett 447: 109-114, 2008.

5. Culley DJ, Baxter MG, Yukhananov R and Crosby G: Long-term impairment of acquisition of a spatial memory task following isoflurane-nitrous oxide anesthesia in rats. Anesthesiology 100: 309-314, 2004

6. Satomoto M, Satoh Y, Terui K, et al: Neonatal exposure to sevoflurane induces abnormal social behaviors and deficits in fear conditioning in mice. Anesthesiology 110: 628-637, 2009. 
7. Haseneder R, Kratzer S, von Meyer L, Eder M, Kochs E and Rammes G: Isoflurane and sevoflurane dose-dependently impair hippocampal long-term potentiation. Eur J Pharmacol 623: 47-51, 2009.

8. Ishizeki J, Nishikawa K, Kubo K, Saito S and Goto F: Amnestic concentrations of sevoflurane inhibit synaptic plasticity of hippocampal CA1 neurons through gamma-aminobutyric acid-mediated mechanisms. Anesthesiology 108: 447-456, 2008

9. Mason LJ, Cojocaru TT and Cole DJ: Surgical intervention and anesthetic management of the patient with Parkinson's disease. Int Anesth Clin 34: 133-150, 1996.

10. Ng M, Roorda RD, Lima SQ, Zemelman BV, Morcillo P and Miesenböck G: Transmission of olfactory information between three populations of neurons in the antennal lobe of the fly. Neuron 36: 463-474, 2002.

11. Zimprich A, Biskup S, Leitner P, et al: Mutations in LRRK2 cause autosomal-dominant parkinsonism with pleomorphic pathology. Neuron 44: 601-607, 2004.

12. Liu Z, Wang X, Yu Y, et al: A Drosophila model for LRRK2-linked parkinsonism. Proc Natl Acad Sci USA 105 2693-2698, 2008.

13. Choi DC, Chae YJ, Kabaria S, et al: MicroRNA-7 Protects against 1-Methyl-4-Phenylpyridinium-Induced Cell Death by Targeting RelA. J Neuroscience 34: 12725-12737, 2014.

14. White KE, Humphrey DM and Hirth F: The dopaminergic system in the aging brain of Drosophila. Front Neurosci 4: 205, 2010.

15. Braak H and Del Tredici K: Invited Article: Nervous system pathology in sporadic Parkinson disease. Neurology 70 1916-1925, 2008.

16. Obeso JA, Rodriguez-Oroz MC, Goetz CG, et al: Missing pieces in the Parkinson's disease puzzle. Nat Med 16: 653-661, 2010.

17. Friggi-Grelin F, Coulom H, Meller M, Gomez D, Hirsh J and Birman S: Targeted gene expression in Drosophila dopaminergic cells using regulatory sequences from tyrosine hydroxylase. J Neurobiol 54: 618-627, 2003.

18. Ueno K, Naganos S, Hirano Y, Horiuchi J and Saitoe M: Long-term enhancement of synaptic transmission between antennal lobe and mushroom body in cultured Drosophila brain. Physiology 591:287-302,2013

19. D'Souza RD, Parsa PV and Vijayaraghavan S: Nicotinic receptors modulate olfactory bulb external tufted cells via an excitation-dependent inhibitory mechanism. J Neurophysiology 110: 1544-1553, 2013.

20. Ran D, Cai S, Wu H and Gu H: Di (2-ethylhexyl) phthalate modulates cholinergic mini-presynaptic transmission of projection neurons in Drosophila antennal lobe. Food Chem Toxicol 50: 3291-3297, 2012.

21. Brand AH, Perrimon N: Targeted gene expression as a means of altering cell fates and generating dominant phenotypes. Development 118:401-15, 1993

22. Gu H and O'Dowd DK: Cholinergic synaptic transmission in adult Drosophila Kenyon cells in situ. J Neurosci 26: 265-272, 2006.

23. Gu H and O'Dowd DK: Whole cell recordings from brain of adult Drosophila. J Vis Exp (6): 248, 2007.

24. Yan Y, Yang Y, You J, et al.: Permethrin modulates cholinergic mini-synaptic currents by partially blocking the calcium channel. Toxicol Lett 201: 258-263, 2011
25. Venderova K, Kabbach G, Abdel-Messih E, et al: Leucine-Rich Repeat Kinase 2 interacts with Parkin, DJ-1 and PINK-1 in a Drosophila melanogaster model of Parkinson's disease. Hum Mol Genet 18: 4390-4404, 2009

26. Tai Y, Chen L, Huang E, et al.: Protective effect of alpha-synuclein knockdown on methamphetamine-induced neurotoxicity in dopaminergic neurons. Neural regeneration research 9: 951-958, 2014 Feany MB and Bender WW: A Drosophila model of Parkinson's disease. Nature 404: 394-398, 2000.

27. Dauer W and Przedborski S: Parkinson's disease: mechanisms and models. Neuron 39: 889-909, 2003

28. Forno LS: Neuropathology of Parkinson's disease. J Neuropathol Exp Neurol 55: 259-272, 1996.

29. Mouradian MM: Recent advances in the genetics and pathogenesis of Parkinson disease. Neurology 58: 179-185, 2002.

30. Yamasaki M, Hashimoto K and Kano M: Miniature synaptic events elicited by presynaptic $\mathrm{Ca}^{2+}$ rise are selectively suppressed by cannabinoid receptor activation in cerebellar Purkinje cells. J Neurosci 26: 86-95, 2006.

31. Zhang J, Yang Y, Li H, Cao J and Xu L: Amplitude/frequency of spontaneous mEPSC correlates to the degree of long-term depression in the CA1 region of the hippocampal slice. Brain Res 1050: 110-117, 2005.

32. Talantova M, Sanz-Blasco S, Zhang X, et al: A $\beta$ induces astrocytic glutamate release, extrasynaptic NMDA receptor activation, and synaptic loss. Proc Natl Acad Sci USA 110: E2518-E2527, 2013.

33. Yasuyama K, Meinertzhagen IA and Schürmann FW: Synaptic organization of the mushroom body calyx in Drosophila melanogaster. J Comp Neurol 445: 211-226, 2002.

34. Su H and O'Dowd DK: Fast synaptic currents in Drosophila mushroom body Kenyon cells are mediated by alpha-bungarotoxin-sensitive nicotinic acetylcholine receptors and picrotoxin-sensitive GABA receptors. J Neurosci 23: 9246-9253, 2003.

35. Simkus CR and Stricker C: The contribution of intracellular calcium stores to mEPSCs recorded in layer II neurones of rat barrel cortex. J Physiol 545: 521-535, 2002.

36. Kato R, Tachibana K, Nishimoto N, et al: Neonatal exposure to sevoflurane causes significant suppression of hippocampal long-term potentiation in postgrowth rats. Anesth Analg 117: $1429-1435,2013$

37. Shaltiel-Karyo R, Davidi D, Menuchin Y, et al: A novel, sensitive assay for behavioral defects in Parkinson's disease model Drosophila. Parkinsons Dis 2012: 697564, 2012.

38. Kwon Y, Kim JW, Ho Y, et al: Analysis of antagonistic co-contractions with motorized passive movement device in patients with parkinson's disease. Bio-medical materials and engineering 24: 2291-2297, 2014

39. Oxenkrug GF: The extended life span of Drosophila melanogaster eye-color (white and vermilion) mutants with impaired formation of kynurenine. J Neural Transm 117: 23-26, 2010.

40. Jenner P and Olanow CW: The pathogenesis of cell death in Parkinson's disease. Neurology 66: S24-S36, 2006.

41. Reynolds NP, Soragni A, Rabe M, et al: Mechanism of membrane interaction and disruption by $\alpha$-synuclein. J Am Chem Soc 133: 19366-19375, 2011.

42. Rajput A, Dickson DW, Robinson CA, et al: Parkinsonism, Lrrk2 G2019S, and tau neuropathology. Neurology 67: 1506-1508, 2006.

43. Ross OA, Toft M, Whittle AJ, et al: Lrrk2 and Lewy body disease. Ann Neurol 59: 388-393, 2006. 